\title{
Environmentally Driven Aggregate Façade Systems
}

\author{
Pablo Cabrera Jauregui $\left({ }^{\otimes}\right)$ \\ School of Architecture + Design, Virginia Polytechnic Institute and State University, 811 4th \\ Street NW, Unit 620, Washington, D.C. 20001, USA \\ pcabreraevt.edu
}

\begin{abstract}
Even though computer simulation of environmental factors and manufacturing technologies have experienced a fast development, architectural workflows that can take advantage of the possibilities created by these developments have been left behind and architectural design processes have not evolved at the same rate. This paper presents a design to fabrication workflow that explores data driven design to improve performance of facades, implementing for this purpose computational tools to handle environmental data complexity and proposes robotic fabrication technologies to facilitate façade components fabrication.
\end{abstract}

Keywords: Design computation $\cdot$ Simulation $\cdot$ Fabrication $\cdot$ Design robotics

\section{Introduction}

Advances in the computer simulation of environmental variables such as light, wind, and sound due to significant growth in the computing power of the day-to-day tools available to designers are expanding the scope of variables that affect design decisions, revealing like never before natural conditions that had remained hidden because of the means of representation traditionally employed by architects to represent their buildings and, consequently, are connecting architecture to the natural sciences (Peters and Peters 2018).

Architectural designs that respond objectively to these environmental constraints are increasingly complex (Schwitter 2005), both in design processes and formal manifestations. The design process is oriented toward performance, but it is important to distinguish two types of performance in architecture: "the kind that can be exact and unfailing in its predictions of outcomes, and the kind that anticipates what is likely, given the circumstantial contingencies of built work. The first sort is technical and productive, the second contextual and projective. There is no need to rank these two in a theory of architectural performance; important instead is grasping their reciprocity and joint necessity" (Leatherbarrow 2009, p. 18). In the context of this paper, performance is understood as the second category.

On the other hand, formal complexity is pushing the boundaries of construction and demanding new means of fabrication. Computer-aided manufacturing processes are increasingly being employed in the fabrication of complex forms, from small prototypes 
to large architectural components, due to increases in the availability and versatility (Willmann et al. 2018) of technologies that until a few decades ago, were the exclusive domain of engineers, such as industrial robots. This versatility plays an important role in the adoption of fabrication technologies in the design and architecture fields.

Even though computer simulations of environmental factors and fabrication technologies have developed rapidly, architectural workflows that take advantage of them have been left behind, and architectural design processes have not evolved at the same rate. Despite some interesting proposals, this is an important area of research that is yet to be fully explored, and now is a good opportunity to create such an integral workflow, from design to fabrication (Hauck and Bergnin 2017), that can negotiate computational frameworks, environmental simulation, and fabrication.

According to the U.S. Green Building Council's "Buildings and Climate Change" (n.d.), the commercial and residential building sector produces $39 \%$ of carbon dioxide emissions in the United States, more than any other sector. Most of these emissions come from the combustion of fossil fuels to provide heating, cooling, and lighting. If embodied emissions, which are the first emissions generated from building materials, products, and construction processes, are taken into account, another problem surfaces. Currently, about 5.7 billion square feet of new buildings are erected in the U.S. every year, and their embodied emissions amount to around 300 million metric tons per year (Strain 2016).
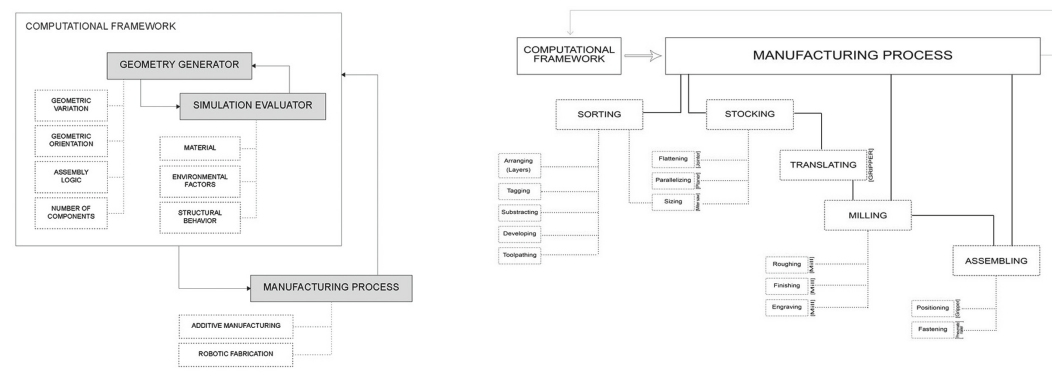

Fig. 1. Independent variables such as materials, environmental conditions, and structural behavior were taken as a framework in which workflow instances were generated based on dependent variables such as geometry, orientation, and assembly logic. Each design experiment explored a specific material system as a means of fabrication and as a manufacturing constraint.

It is not only the performance of the buildings but their materials and construction processes that need to be more energy efficient and climate friendly. At present there is a disconnect between the performance optimization and the fabrication of buildings and their components. This paper explores workflows designed to reconcile this disconnect by proposing new design processes, material systems, and fabrication methods with the aim of moving toward improved performance. The assumption is that by making the built environment more energy efficient and climate friendly, the building sector can play a major role in reducing the threat of climate change. Through immersive case studies focusing on fabrication, we propose workflows that explore data-driven design to improve the performance of facades, implementing for this purpose computational tools 
to handle complex environmental data and proposing robotic fabrication technologies to facilitate façade-component fabrication.

\subsection{Design Experiment: Timber Façade}

The first case study was developed as part of the Eco-Park Learning Center project, a collaboration between the Prince William County Solid Waste Division and the Center for Design Research in the School of Architecture + Design at Virginia Tech. Visitors to the Eco-Park Learning Center are taught about a range of alternative energy sources, including solar, wind, and methane.

(a)

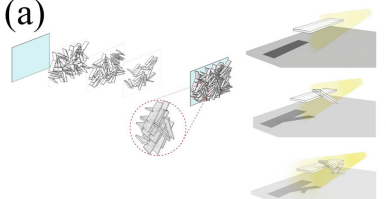

(d)

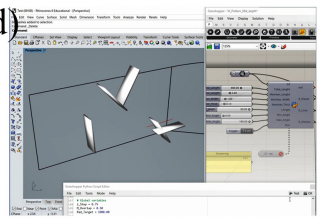

$(\mathrm{g})$

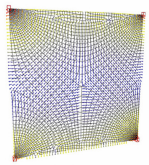

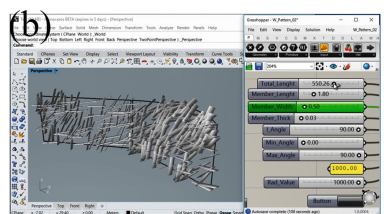

$(\mathrm{e})$

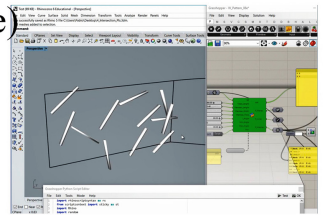

(h)

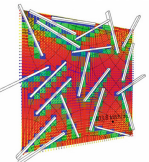

(c)

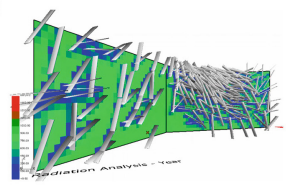

(f)

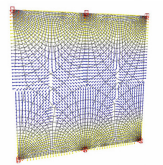

(i)

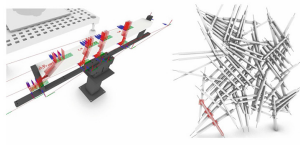

Fig. 2. From top left Figures (a) through (i) showing steps of the computational framework including geometry generation, environmental and structural simulation and robot toolpathing.

\subsubsection{Aim}

The shading screen for the PWC project was conceived of as a way to reduce buildings' solar exposure by means of a facade shading device made from the recycled wood commonly used in construction scaffolding (Fig. 2a).

The aim in the first part of the research process is to develop a computational framework that can produce instances of a geometric system informed by material constraints and environmental considerations. The aim of the second part is to explore the fabrication of the system's assembly logic based on notches, to understand the limitations of the fabrication and the opportunities for a robotic mill fabrication workflow to produce wood joints, and to inform the construction of the system as a fabrication constraint. 


\subsubsection{Computational Framework}

Within the computer-aided design environment Rhinoceros, a custom Python script was developed that could generate components through a bottom-up, additive process in which the final morphology is a result of the initial-condition rules.

Figure $2 b$ shows all the variables implemented to control the geometry of the screen. The addition of each wood member is linked to a yearly direct-incidence solar radiation simulation based on the Ladybug plugin (Fig. 2c). The algorithm implemented in the script places a new member at each point the simulation determines to have the highest exposure to the sun, reducing the global facade exposure.

Figure $2 \mathrm{~d}$ shows the assembly logic of the system. Notches are added between intersecting members so that every time a new member intersects an older one, it jumps a layer outward by a proportion of its width and a scale-down variable. This controls the cross-section of every new layer. The potential here is for the fade-out the facade as it continues adding layers by cutting the original wood piece in halves, quarters, and so forth. For the studied prototypes, the same size of cross-sections was used in all facade layers.

It is also possible to specify as a variable in the computational framework the minimum number of intersections before a new member jumps out a layer. Figure 2e shows case of two intersections.

\subsubsection{Manufacturing}

The fabrication workflow involves several manufacturing processes, as shown in Fig. 1. To test the workflow, a mockup instance of the computational framework was developed for fabrication.

The generation process was informed using the direct solar radiation on the position for the components, and using computational finite-element structural analysis to determine the stress lines and align the angles of each member.

Figure $2 \mathrm{f}$ and Fig. $2 \mathrm{~g}$ show different support cases, displayed as wire boxes, which generate different stress line patterns. Members are aligned to these patterns so as to provide material continuity (Fig. 2h). The mockup screen is $8^{\prime} \times 8^{\prime}$ and composed of three layers of members with $2^{\prime} \times 4^{\prime}$ cross sections and a length of 3 feet, responding to spring-summer solar radiation.

A sorting procedure in the form of a Python script was developed that arranges layers and parts and also tags, subtracts, and develops the members generated by the computational framework (Fig. 2i). A second custom Python script takes the output of the sorting procedure and automatically generates toolpaths for the robot (Fig. 3a).

\section{Robotic Fabrication}

The fabrication of the mockup made from $2^{\prime} \times 4^{\prime}$ pine members took place in Boston as part of a residency at the Autodesk BUILD Space (Fig. 3b), a research and development workspace focused on innovation in architecture, engineering, and construction. The residency period was divided into two parts, one to develop, produce, and test tools for the fabrication process, and one for the actual fabrication of the complete prototype.

A manufacturing cell composed of an ABB robot model IRB 4600 with a Spindle tool and a safety guard was used for the fabrication (Fig. 3c). Because the industrial 
(a)
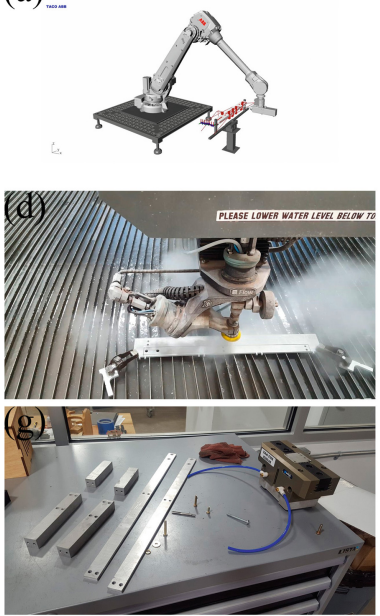
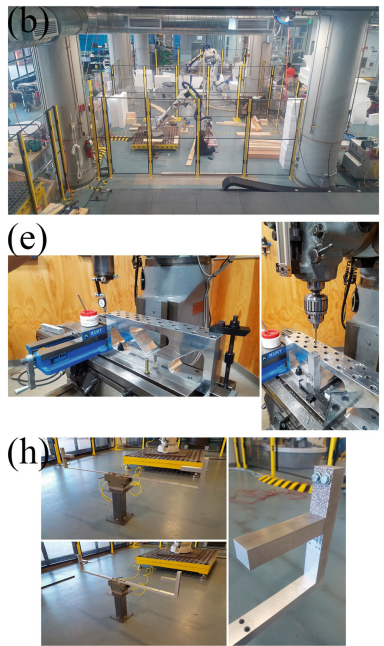
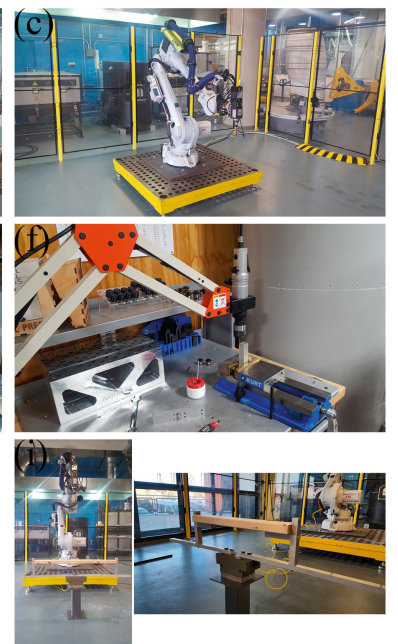

Fig. 3. From top left Figures (a) through (i) showing simulation of robot toolpaths, the robot employed for fabrication at Autodesk BUILD Space, and custom gripper production and early tests.

robot is a versatile machine, it is open-ended, meaning that in every fabrication project involving robots, all the tools needed must be designed and fabricated and from a design point of view. New fabrication skills must also be acquired, from operating advanced $\mathrm{CNC}$ equipment to precision machining. In the case of the fabrication of the mockup, the focus was on the work holding.
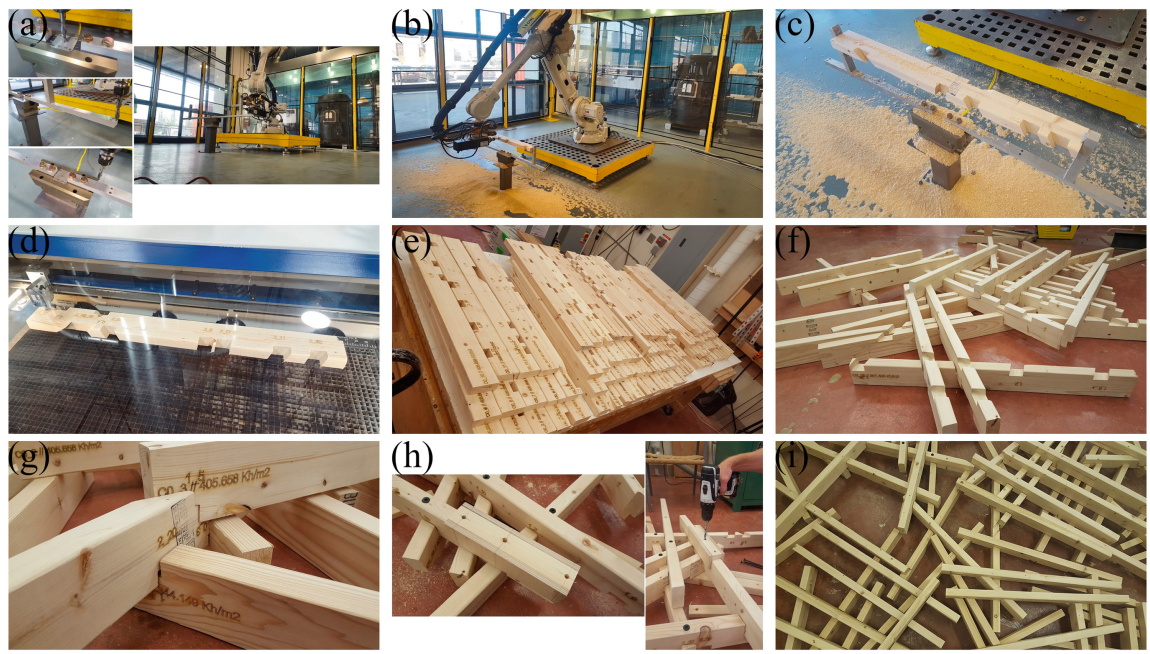

Fig. 4. From top left Figures (a) through (i) showing robot calibration, milling and labeling of a façade component, and assembly of screen. 
Fingers to hold the wooden pieces were designed and produced based on a pneumatic gripper. Parts were waterjet-cut from 1/4-inch aluminum plate (Fig. 3d) and drilled using a Bridgeport (Fig. 3e). This is an especially critical step for tolerances, so calibration equipment was used to ensure perpendicularity between the gripper's component parts. Finally, the parts were tapped using a Haas machine (Fig. 3f). Figure $3 \mathrm{~g}$ shows the custom-made parts that compose the work holding.

After the work holding was assembled (Fig. 3h), two problems were detected. The longest aluminum parts were too thin for their length and tended to vibrate when forces were applied to their extremes. These were replaced with $3 / 4$-inch aluminum parts. The second problem had to do with the grip capacity of the fingers contacting the wood pieces. When working on naked aluminum, the wooden pieces tended to slip, so low-grain sandpaper was added as a surface contact (Fig. 3i).

Because the floor of the manufacturing cell was not perfectly horizontal, a calibration procedure was established to read the inclination of the work holding in relation to the robot (Fig. 4a). The toolpath-generator script has the flexibility to read test coordinate points taken from the physical gripper, so the toolpaths generated for the robot can deal with this discrepancy between the digital and physical worlds.

Figure $4 \mathrm{~b}$ shows the milling process, and Fig. $4 \mathrm{c}$ shows the final wooden piece after milling. To aid in the assembly process, information generated by the sorting scriptsuch as component name, intersecting component at each notch, and direct-incidence solar radiation value at the time of the component being added-was engraved on every component (Fig. 4d) using a laser cutter machine.

The mockup was assembled in Blacksburg at the woodshop facilities of Virginia Tech (Figs. 4e, 4f, 4g). While the notch logic helped secure every piece in place, $4 \frac{1}{2} 2$-inch structural screws were used to fasten them locally using power screwdrivers (Fig. 4h). Figure 4i and Fig. 5d shows the completed mockup.

\subsubsection{Results and Findings}

As Fig. 5 b shows, the computational framework plus the simulation evaluator can reduce the facade solar exposure in the PWC project from $890.76 \mathrm{Kwh} / \mathrm{m}^{2}$ to $369.32 \mathrm{Kwh} / \mathrm{m}^{2}$ by adding a screen on the southeast-facing facade.

The assembly logic based on notches (Fig. 5a) works for positioning the components. A tolerance of $2 \mathrm{~mm}$ was introduced during the milling process to account for calibration errors and material changes, such as wood swelling due to humidity. For this reason, structural screws were also employed to secure each notch.

As a manufacturing proof of concept, part of the facade was fabricated at the Autodesk BUILD Space in Boston and later displayed at the ICFF exhibition in New York (Fig. 5c). 

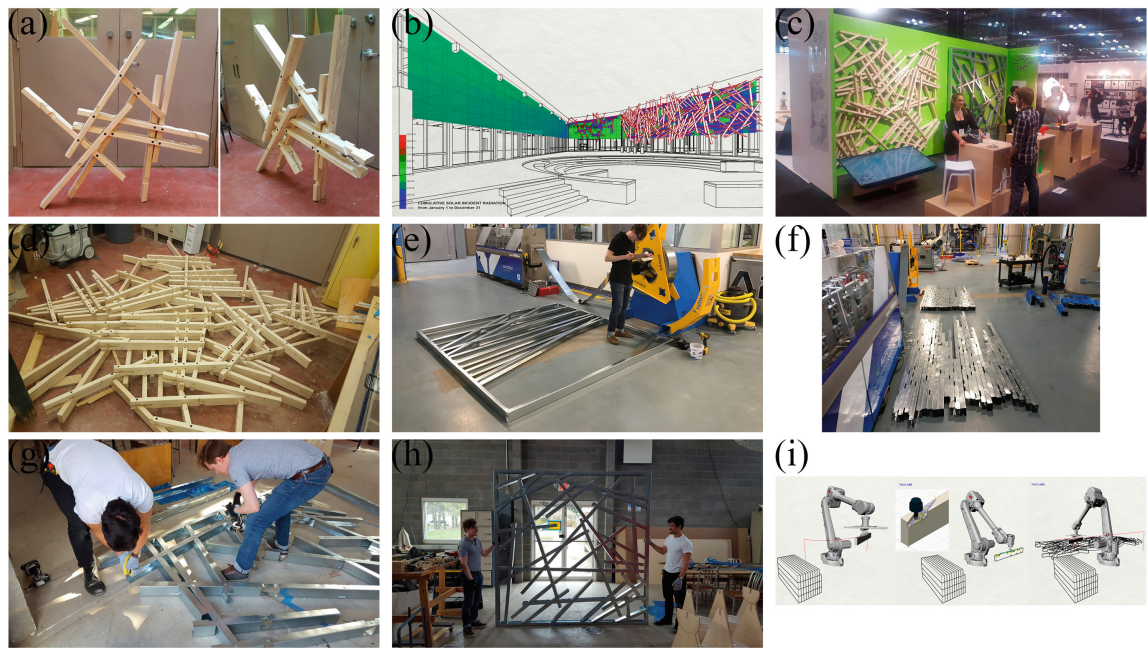

Fig. 5. From top left Figures (a) through (i) showing assembled parts of the screen, the architectural target façade, the metal façade fabrication and assembly process, and the proposed fully automated manufacturing scenario.

\subsection{Design Experiment: Metal Façade}

A parallel fabrication method was tested to explore the consistency of the workflow from design to production. The outcome of the computational framework and the simulation evaluator was diverted to another fabrication method.

\subsubsection{Aim}

The objective of this experiment was to test the viability of an alternative fabrication method for the outcome of the computational framework and simulation evaluator used for the shading screen.

\subsubsection{Computational Framework}

In the computational framework developed for the Timber Façade design experiment, a Python script was hooked up to collect center-line geometric information of the facade components. The number of layers composing the façade was kept at zero. A custom Grasshopper plugin running inside the Rhinoceros CAD environment was then used to export the comma-separated value files, which were inputted into the Howick frame machine (Fig. 5e).

\subsubsection{Manufacturing}

The generated morphology was fabricated using a Howick frame machine, which can bend, cut, and punch out thin metal rolls. Figure 5f and Fig. $5 \mathrm{~g}$ show the assembly process and the final metal piece. 


\section{Light-Gauge Steel Framing}

According to Howick's manufacturer information, steel framing machines place all punching and fixing holes using accurate computer control. This allows the frames to be manufactured with high precision and to be self-locating and jigging. All the frame components are produced quickly, with the location dimples and pre-punched screw and rivet holes ready for assembly and clearly marked. No further cutting or post-processing work is needed, so low-skilled local labor can be used to assemble the buildings with little supervision.

\subsubsection{Results and Findings}

The viability of an alternative fabrication method was demonstrated (Fig. 5h). The addition of custom code made it possible to expand the material alternatives in which the outcome of the computational framework could be fabricated.

\section{Conclusion}

Contributions of this work are weighted heavily toward the process rather than the products, and central to this is multivariable design to fabrication workflow that interrelates a computational framework, environmental performance simulation, and computer-aided manufacturing. A sub-process of this workflow is a form-finding computational strategy for an environment-driven facade in the form of Python scripts. Instances of the workflow have been used to fabricate prototypes as a proof of concept of the process.

From the case studies presented here, is possible to state that a robotics-based fabrication method informed by a multi-variable computational framework and a simulation evaluator integrated into a design-to-fabrication workflow is feasible. Instances of this workflow, such as the shading screen for PWC, show a responsiveness to environmental conditions that stems from the logic defined in the workflow. From a representational point of view, when environmental data are made visible with numbers, as in the solar radiation simulation on the shading screen, they can be integrated as a design variable because the designer is objectively aware of their influence in the same way she is aware of a drawing or an area schedule. From a computational point of view, the use of scripts and subroutines for environmental data processing allow larger data quantities to be considered, such as the yearly solar radiation results used in the shading screen. Finally, from a manufacturing point of view, the versatility of industrial robots allows them to be used in a wide range of fabrication scenarios. The definition of a tool through the fabrication process is what gives it specificity, and complex forms can be fabricated with a well-designed robot tool.

\section{Future Research}

One promising line of research would be the consolidation of separate manual processes (Fig. 1) into a comprehensive robotic fabrication workflow, in which a continuous robotic process manufactures the façade, from material sourcing to assembly (Fig. 5i). Integrated design-to-fabrication workflows can also help the building industry become more energy 
efficient and climate friendly on two different scales. On a material scale, embodied carbon emissions can be reduced by the use of recycled or self-grown materials. In the case of the shading screen, for instance, the workflow proposes as material variables both rescued timber from construction scaffolding and pine wood from responsibly managed forests that provide environmental, social, and economic benefits. During the manufacturing process, embodied carbon emissions can be lowered through the use of more efficient prefabrication dry methods. For instance, in the case of the shading screen, a manufacturing scenario employing the Howick steel framing machine was proposed because of its use of light-gauge steel, which is a modern form of building that has been proven to reduce environmental impact.

This leads to interesting questions about the role of the architect. The increasing availability of advanced manufacturing technologies, means the profession is returning to the consideration of construction and manufacturing as a part of the design process, and this returns to the architect control over the fabrication of her work, blurring the line between design and construction that was artificially created by modernism. But it also blurs the traditional role of the architect, confronting her with a multidisciplinary set of new skills and knowledge.

\section{References}

Buildings and Climate Change (n.d.). U.S. Green Building Council. http://www.usgbc.org

Hauck, A., Bergnin, M.: The triumph of the turnip. In: Sheil, B., Menges, A., Glynn, R., Skavara, M. (eds.) Fabricate 2017, pp. 16-21. UCL Press, London (2017)

Leatherbarrow, D.: Architecture Oriented Otherwise. Princeton Architectural Press, New York (2009)

Peters, B., Peters, T.: Computing the Environment: Digital Design Tools for Simulation and Visualization of Sustainable Architecture. Wiley, Hoboken (2018)

Schwitter, C.: Engineering complexity: performance-based design in use. In: Kolarevic, B., Malkawi, A. (eds.) Performative Architecture: Beyond instrumentality, pp. 113-122. Spon Press, New York (2005)

Strain, L.: Building Materials and the Time Value of Carbon (2016). https://www.buildinggreen. com

Willmann, J., Block, P., Byrne, K., Hutter, M., Schork, T.: New scientific frontiers. In: Willmann, J., Block, P., Byrne, K., Hutter, M., Schork, T. (eds.) Robotic Fabrication in Architecture, Art and Design 2018, pp. ix-xiv. Springer, Switzerland (2018) 
Open Access This chapter is licensed under the terms of the Creative Commons Attribution 4.0 International License (http://creativecommons.org/licenses/by/4.0/), which permits use, sharing, adaptation, distribution and reproduction in any medium or format, as long as you give appropriate credit to the original author(s) and the source, provide a link to the Creative Commons license and indicate if changes were made.

The images or other third party material in this chapter are included in the chapter's Creative Commons license, unless indicated otherwise in a credit line to the material. If material is not included in the chapter's Creative Commons license and your intended use is not permitted by statutory regulation or exceeds the permitted use, you will need to obtain permission directly from the copyright holder.

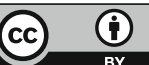

